\title{
Predictive factors of postoperative complications after cytoreductive surgery for ovarian cancer
}

\author{
Atallah D, Sarkis Y, Richa F, Sleilaty G, Moubarak M, El Kassis N, Chahine G, Chalhoub V \\ Hôtel-Dieu de France University Hospital, Saint Joseph University, Beirut, Lebanon
}

\section{BACKGROUND/AIM}

Ovarian cancer is the leading cause of death from gynecological cancer for women. The standard treatment consists of an extensive cytoreductive surgery followed with adjuvant chemotherapy. This study aims to identify the common postoperative complications as well as to define predictive factors of their

\section{METHODS}

This prospective study was conducted at Hôtel-Dieu University Hospital in Lebanon between October 2017 and October 2018. All patients older than 18-year-old who underwent cytoreductive surgery for ovarian cancer were followed up from the postoperative admission in the intensive care unit till discharge from hospital or at least for $\mathbf{3 0}$ days. Correlations between perioperative characteristics and complications were searched and analyzed.

\section{RESULTS}

- 40 patients were included. The mean age was 55 years old. The mean surgical complexity score was 5.

- Major complications have occurred in $32 \%$ of cases. They were associated with neoadjuvant chemotherapy $(p=$ 0.009), elevated surgical complexity $(p=0.037)$, need for intraoperative transfusion and stay at intensive care unit more than 48 hours $(p=0.05)$.

- Complications were infectious, hemodynamic, pulmonary, digestive and surgical.

- Need for parenteral nutrition was significantly correlated with longer operative time and neoadjuvant chemotherapy. No correlation was found between occurrence of complications and the following parameters: age, stage, APACHE II score, Charlston Comorbidity index and preoperative albuminemia.

\begin{tabular}{|c|c|c|c|}
\hline Complications & Independant variables & $\begin{array}{l}\text { P- } \\
\text { value }\end{array}$ & $\begin{array}{l}\text { OR + confidence } \\
\text { interval }\end{array}$ \\
\hline Severe & High surgical complexity & 0.037 & $4.6(1.10-19.20)$ \\
\hline \multirow[t]{3}{*}{ Complications } & Neoadjuvant chemotherapy & 0.009 & $7(1.59-30.60)$ \\
\hline & Intraoperative blood transfusion & 0.025 & - \\
\hline & Stay in ICU > $48 \mathrm{hrs}$ & 0.05 & - \\
\hline Transfusion of & Neoadjuvant Chemotherapy & 0.015 & $7(1.30-37.70)$ \\
\hline $\begin{array}{r}\text { Packed RBCs in } \\
\text { ICU }\end{array}$ & Intraoperative transfusion & 0.05 & $4.125(1.01-18.52)$ \\
\hline Need for & Neoadjuvant chemotehrapy & 0.011 & I3.8 (I.46-|30.07) \\
\hline $\begin{array}{l}\text { parenteral } \\
\text { nutrition }\end{array}$ & Operative duration & 0.001 & \\
\hline \multirow[t]{3}{*}{ Re-admission } & High surgical complexity & 0.037 & $4.6(1.038-20.38)$ \\
\hline & Need for intraop. platelets transfusion & 0.015 & $16(1.53-166.53)$ \\
\hline & Intubated during transfer to ICU & 0.038 & - \\
\hline Need for & Neodajuvant chemotherapy & 0.05 & - \\
\hline vasopressors & $\begin{array}{l}\text { Need for intraopertive platelets } \\
\text { transfusion }\end{array}$ & 0.036 & $22.667(1.56-328.94)$ \\
\hline $\begin{array}{l}\text { Hemodynamic } \\
\text { complications }\end{array}$ & Stade III-IV & 0.019 & $9.5(3.76-24.00)$ \\
\hline Infectious & Platelets transfusion (intraoperatively) & 0.049 & $1.313(1.03-1.66)$ \\
\hline \multirow{2}{*}{ complications } & Packed RBCs transfusion & 0.017 & $6.909(1.23-38.5 I)$ \\
\hline & Intubated during transfer to ICU & 0.049 & $1.313(1.03-1.66)$ \\
\hline \multirow[t]{2}{*}{ Pneumonia } & High surgical complexity & 0.022 & - \\
\hline & Platelets transfusion (intraoperatively) & 0.036 & $22.667(1.56-328.94)$ \\
\hline Urinary infection & High surgical complexity & 0.05 & $6.5(1.99-42.31)$ \\
\hline \multirow{3}{*}{$\begin{array}{l}\text { Acute renal } \\
\text { insufficiency }\end{array}$} & Neoadjuvant chemotherapy & 0.007 & - \\
\hline & $\begin{array}{l}\text { Complexité chirurgicale élevée aux } \\
\text { high surgical complexity }\end{array}$ & 0.022 & $13.5(|.3|-|38.6|)$ \\
\hline & Platelets transfusion (intraoperatively) & 0.009 & $24.75(2.50-244.09)$ \\
\hline \multirow[t]{2}{*}{ Nausea/vomiting } & Preoperative ascites & 0.015 & $5.867(1.30-26.32)$ \\
\hline & High surgical complexity & 0.043 & $4.2(1.00-17.57)$ \\
\hline
\end{tabular}

Table I. significative correlations

\section{CONCLUSION}

Cancer stage, neoadjuvant chemotherapy, high surgical complexity, need for transfusions, delayed extubation and stay at intensive care unit more than 48 hours were predictive factors of higher postoperative morbidity in patients receiving cytoreductive surgery for ovarian cancer. 